\title{
Quantifying sustainable control of inventory systems with non-linear backorder costs
}

\author{
Lina Johansson $^{1}$ - Fredrik Olsson ${ }^{1}$
}

Published online: 30 May 2017

(C) The Author(s) 2017. This article is an open access publication

\begin{abstract}
Traditionally, when optimizing base-stock levels in spare parts inventory systems, it is common to base the decisions either on a linear shortage cost or on a certain target fill rate. However, in many practical settings the shortage cost is a non-linear function of the customer waiting time. In particular, there may exist contracts between the spare parts provider and the customer, where the provider is obliged to pay a fixed penalty fee if the spare part is not delivered within a certain time window. We consider a two-echelon inventory system with one central warehouse and multiple local sites. Focusing on spare parts products, we assume continuous review base stock policies. We first consider a fixed backorder cost whenever a customer's time in backorder exceeds a prescribed time limit, second a general non-linear backorder cost as a function of the customer waiting time, and third a time window service constraint. We show from a sustainability perspective how our model may be used for evaluating the expected $\mathrm{CO}_{2}$ emissions associated with not satisfying the customer demands on time. Finally, we generalize some known inventory models by deriving exact closed form expressions of inventory level distributions.
\end{abstract}

Keywords Inventory - Multi-echelon · Non-linear backorder cost - Time window service constraint $\cdot$ Sustainability

\section{Introduction and literature review}

In many industries spare parts and aftersales in general are big business (Cohen et al. 2006). For example, the US automotive aftermarket was estimated to be worth $\$ 188.6$ billion in 2007 (US Automotive Parts Industry Annual Assessment 2009). Another example is the

\footnotetext{
$\bowtie$ Fredrik Olsson

fredrik.olsson@iml.lth.se

Lina Johansson

lina.johansson@iml.lth.se

1 Department of Industrial Management and Logistics, Lund University, P.O. Box 118, 22100 Lund, Sweden
} 
aviation industry, which stocks spare parts for several billion US-dollars (Harrington 2007). However, although it may be very costly to invest in expensive spare parts, it is crucial to have spare parts available when needed. Obviously, delays and downtimes of bottleneck production equipment may be very costly. A particularly striking example is in the oil rig industry, where the production downtime on an oil rig may incur a cost of $\$ 200,000$ per day (Turban 1988). For a general overview of models for spare parts inventory control see, e.g., Basten and van Houtum (2014).

We consider a two-echelon inventory system with $N$ locations and one central warehouse. All locations and the warehouse apply $(S-1, S)$ policies (i.e., continuous review base stock control), which is reasonable for low demand items such as spare parts. This paper extends the literature on spare parts inventory control in several new directions. Three different backorder/service level structures are investigated in this paper. In more precise terms, we consider: (1) a model with piecewise constant backorder costs, (2) a model with general non-linear backorder costs, and finally (3) a model with time window service constraints. The motivation for considering these three cases stems from collaboration and discussions with industry, and the state of the current research frontier. In connection with case (1), one main focus is also to develop an approach that can be used in order to explicitly evaluate the expected $\mathrm{CO}_{2}$ emissions associated with not satisfying the customer demands on time. We provide an exact analysis for all cases considered.

Considering case (1), all unsatisfied demands are backordered and the customers at each location $i$ are satisfied if their demands are met directly or after an acceptable waiting time $\omega_{i}$. That is, if a customer receives the requested unit within this acceptable waiting time, no penalty cost is incurred. If a customer, on the other hand, has to wait longer than this given time limit, a considerable fixed backorder/penalty cost has to be paid. This backorder cost is independent of the additional waiting time exceeding $\omega_{i}$. This particular scenario is quite common in many practical settings. One example is Tetra Pak Technical Services which provides customers in the dairy industry with spare parts for packaging machines. In such a case, having to wait for a critical spare part means that the production process is halted. While a short downtime may be acceptable, it is more crucial if the customer at the production site has to wait for the spare part longer than a certain critical time limit. Then, if the critical time limit is violated, the whole batch of the dairy product must be discarded due to the perishable nature of the product. In situations like this one, it is quite common that there exists a service agreement (or contract) in which it is stipulated that the service provider should pay a fixed penalty if the spare part is not delivered within a certain time window. In this case, the fixed penalty cost is directly associated with the cost of discarding the batch of the dairy product (and to some extent also the set up cost for a new batch). Other similar examples can be found in the agriculture sector, where a whole (or parts of) harvest may be lost if the harvest-machines are down due to missing critical spare parts. Similarly as in the case with dairy products, a lost harvest incur a large, well defined, fixed cost.

Another main contribution of this paper is to take a first step in quantifying how decision rules for the logistics system affect the expected $\mathrm{CO}_{2}$ emissions from a production waste perspective. As noted in Marklund and Berling (2017), there are few models that include emissions associated with not satisfying customer demand in a timely fashion. Marklund and Berling (2017) argue that this is particularly accentuated in the distribution of spare parts, and they explicitly state: "The parts are often quite small suggesting quite limited emissions associated with transportation, inventory holding and warehousing. However, not delivering them promptly may have serious consequences on costs and emissions". In fact, most literature concerning how to control supply chain systems in order to reduce (or at least quantify) $\mathrm{CO}_{2}$ emissions have primarily focused on transportation issues, see e.g. McKinnon 
(2010) and Alkawaleet et al. (2014). However, it is important to notice that supply chain policy decisions also affect $\mathrm{CO}_{2}$ emissions related to waste at the customer sites. For example, as noted above, a whole production batch of a dairy product may be wasted if the coordination of supply and demand for critical spares is not aligned.

Of course, there may exist cases where other types of backorder cost structures are more plausible than a model with piecewise constant backorder costs. Therefore, in case (2) we generalize the structure of the backorder cost and focus on general non-linear backorder costs as a function of the waiting time. For many companies a quite long waiting time for a critical spare part may be severe, while a relatively short waiting time may not incur very large costs. Hence, although our modeling technique can handle general non-linear backorder costs, we focus on exponentially increasing backorder costs due to its intuitive and appealing features from a practical point of view. Another possible situation may be when the backorder cost is so called S-shaped. This means that the backorder cost as a function of longer waiting times is concave (instead of convex as in the exponential case). We provide a model that can handle all such possible backorder cost structures (as mentioned, the exponential case should be viewed as a concrete example).

In case (3) we consider a service level instead of a model with backorder costs. This service level is defined as the probability of satisfying a customer demand within a certain time window. In all three cases we utilize information about the timing of outstanding orders for the central warehouse and the downstream locations, respectively.

Early studies on continuous review multi-echelon inventory models include, e.g., Sherbrooke (1968), Graves (1985) and Axsäter (1990). Sherbrooke (1968) considers a twoechelon inventory system with multiple local retailers and one central warehouse, all applying $(S-1, S)$ ordering policies where unsatisfied demands are backordered. Given this inventory system, he develops an approximate method (the METRIC approximation), where the real stochastic leadtimes for the retailers are approximated by their mean values. Graves (1985) extends the results from Sherbrooke (1968) by deriving an exact solution procedure. However, one of the main problems is that the solution provided in Graves (1985) is not in closed form, which means that the evaluation of the inventory level probabilities will be approximate (due to necessary truncation of infinite series). In order to ease the computational burden, Graves (1985) also presents corresponding approximate distributions. Given the same inventory system as in Graves (1985), Axsäter (1990) develops a different exact solution procedure. However, similar to Graves (1985), Axsäter (1990) does not either present a closed form solution. Moreover, he does not derive the distribution of the inventory levels for the local retailers. Instead, he derives a recursive solution procedure in order to obtain the total average cost. In this paper, we extend the analysis of Graves (1985) and Axsäter (1990) by deriving closed form solutions of the probability distributions of the inventory levels and the customer waiting times. For more information concerning continuous review multi-echelon inventory systems see, e.g., Axsäter (1993).

The literature on multi-echelon inventory models with time window service levels is relatively limited. Ettl et al. (2000) consider a multi-echelon inventory system with similar service requirements as in our model. They model their inventory system as a $M^{X} / G / \infty$ queueing system, and consider both assembly and distribution structures. Although Ettl et al. (2000) in some aspects consider a more general model than we do, their analysis require assumptions like leadtimes based on the exponential distribution. Another related model is presented in Caggiano et al. (2007), where the problem in Ettl et al. (2000) is extended to a multi-item setting. A limitation of this work is that the acceptable customer waiting times at different sites $\left(\omega_{i}\right.$ in our case) are only allowed to be multiples of the transportation times between different echelons. Another study on multi-echelon inventory models with time 
window service levels is Wong et al. (2007), which deals only with average time window constraints.

One category of papers in the literature that is quite related to our work is inventory models with emergency supply. Two papers that fall into this category are Moinzadeh and Schmidt (1991) and Moinzadeh and Aggarwal (1997). The former studies a single location system, where the location applies an $(S-1, S)$ policy and has the option to choose either a normal order or an emergency order under a given ordering policy. This policy takes all available information regarding the inventory level and timing of outstanding orders into account. Moinzadeh and Aggarwal (1997) extend the work done by Moinzadeh and Schmidt (1991) by considering a base-stock two-echelon inventory system with one warehouse, multiple retailers and an outside supplier with the possibility of emergency orders. Huang et al. (2011) introduces a committed service time, in which it is acceptable for the customer to be served. After this time has passed, the retailers face a backorder cost (per unit and time unit) and has the ability to fill the demand with an emergency order. In a more recent paper, Howard et al. (2015) evaluate an approximate two-echelon spare parts inventory model using pipeline information. In more detail, they consider an inventory system that includes a central warehouse acting as a supplier and a so called support warehouse. The purpose of the support warehouse is to provide emergency orders to the local warehouses, and as a last resort emergency transshipments can be sent directly from the central warehouse. All of these papers mentioned above consider a standard unit backorder cost per time unit. However, in our model we consider general non-linear backorder costs, and in particular piecewise constant backorder costs.

Another related stream of literature concerns lateral transshipments between warehouses, where a local warehouse with no stock on hand can request an item from another local warehouse if needed. Yang et al. (2013) assumes, just as we do, that customers have a certain pre-specified acceptable waiting time limit. Within this time limit, the local warehouse will wait for an incoming uncommitted item. If the waiting time is too long, it will request a lateral transshipment having a shorter, but positive, leadtime. Olsson (2015) studies a similar model as Yang et al. (2013), but uses a backorder cost per unit and time unit instead of a time limit. For a review of papers studying lateral transshipments see, e.g., Paterson et al. (2011).

The literature discussed so far is, in general, based on one-for-one ordering policies, which is also the case we consider in this paper. When instead considering batch-ordering policies in multi-echelon inventory settings with time window service levels, the model complexity will increase considerably. In a single-echelon setting Axsäter (2003b) considers an inventory system with lateral transshipments where the locations apply $(R, Q)$ policies. In Axsäter (2003b) an approximate method is developed that uses information about the residual leadtimes of the items in the system. Other relevant literature concerning batch-ordering policies include, e.g., Axsäter (2000) and Katehakis and Smit (2012).

In the following section we formulate our model in detail. Section 3 presents the solution procedure with the derivation of exact closed form expressions for various performance characteristics. In Sect. 4, cost structures are presented together with cost evaluations. In Sect. 5, we present cost optimization procedures for all three cases considered, and in Sect. 6 numerical examples are presented and discussed. In Sect. 6, we also give an application of how to use the theory developed in order to quantify sustainability measures, such as expected $\mathrm{CO}_{2}$ emissions related to production waste. Some concluding remarks are given in Sect. 7. 


\section{Model formulation}

We consider a two-echelon continuous review inventory model with one central warehouse and $N$ local sites. All transportation times are positive and constant. Customer demands follow independent Poisson processes and occur only at the local sites. Since we focus on spare parts products, we assume that replenishments are made according to base stock ordering policies. All unsatisfied demands are backordered. Furthermore, we assume that backorders at the sites and at the central warehouse are filled according to the FCFS (first come - first served) rule.

In similar models like this one, it is commonly assumed that base stock levels are based on a backorder cost per unit and time unit or a prescribed service level. In those cases a target service level is used, the service level definition is very often the so called fill rate (fraction of demand that can be satisfied directly from stock on hand). However, in this paper, we consider one step function and one general non-linear backorder cost structure, where the cost is a function of the customer's time in backorder. In addition, we also consider a time window based service level.

Let us introduce some notations for parameters and decision variables:

$\lambda_{i}-$ customer arrival rate at local site $i$,

$L_{i}-$ transportation time from the central warehoue to local site $i$,

$L_{0}-$ transportation time from the supplier to the central warehouse,

$h_{i}$ - holding cost per unit and time unit at local site $i$,

$h_{0}-$ holding cost per unit and time unit at the warehouse,

$N$ - number of local sites,

$S_{i}$ - base-stock level at local site $i$,

$S_{0}-$ base-stock level at the warehouse,

We proceed by defining the two cost structures and the time window based service level in more detail. In the subsequent analysis, let $Y_{i}$ denote the time a customer demand is backordered at site $i$. Note that $0 \leq Y_{i} \leq L_{0}+L_{i}$ is stochastic and depends on the basestock levels at site $i$ and the central warehouse.

\section{Performance characteristics}

Define $X_{0}$ as the limiting (i.e., the stationary case) age of the oldest unit at the warehouse not assigned to any waiting customer, where the age is assumed to start when the unit is ordered from the outside supplier. Similarly, let $X_{i}$ be the limiting age of the oldest unit at site $i(i=1, \ldots, N)$ not assigned to any waiting customer, where the age of an item is assumed to start when the unit is ordered from the warehouse. Note that, if the warehouse has zero stock on hand when a site orders a unit, the ordered unit will arrive at the site after $L_{i}+Z$ unit of time, where $Z$ is the stochastic delay at the warehouse. Obviously, we must have $Z=L_{0}-X_{0}$, for $0 \leq X_{0} \leq L_{0}$. Hence, given a stochastic delay of $Z$ units of time at the warehouse, the oldest unit at site $i$ is outstanding if $0<X_{i}<L_{i}+Z$, and in stock if $X_{i} \geq L_{i}+Z$.

Let us proceed by noting that $X_{0} \sim \operatorname{Erlang}\left(\lambda_{0}, S_{0}\right)$ and $X_{i} \sim \operatorname{Erlang}\left(\lambda_{i}, S_{i}\right)$, where $\lambda_{0}=\lambda_{1}+\cdots+\lambda_{N}$. This is the case since $\left(S_{i}-1, S_{i}\right)$ policies are used and that the demand process is pure Poisson at the sites and at the warehouse, which means that the well known 
PASTA-property (Poisson Arrivals See Time Averages) holds. It should be noted that our technique of tracking the ages of the units in the system yields a richer model than the unit tracking methodology first presented in Axsäter (1990). This is the case since the unit tracking methodology in Axsäter (1990) considers only what happens at times of customer arrivals. In our case, we have full information about the ages of the (oldest) units in the system at all times. This means that, unlike Axsäter (1990), it is possible to generalize our model to incorporate decision rules based on events which are not customer arrivals. In any case, we have the density functions, $f_{X_{i}}(t)$, and the distribution functions, $F_{X_{i}}(t)$,

$$
\begin{gathered}
f_{X_{i}}(t)=\lambda_{i}^{S_{i}} e^{-\lambda_{i} t} \frac{t^{S_{i}-1}}{\left(S_{i}-1\right) !}, \quad t \geq 0 \\
F_{X_{i}}(t)=1-\sum_{n=0}^{S_{i}-1} e^{-\lambda_{i} t} \frac{\left(\lambda_{i} t\right)^{n}}{n !}, \quad t \geq 0
\end{gathered}
$$

for $i=0,1, \ldots, N$. Using (1) and $Z=L_{0}-X_{0}$, it is easy to obtain the density of the stochastic delay, $Z$, as

$$
f_{Z}(t)=f_{X_{0}}\left(L_{0}-t\right)=\lambda_{0}^{S_{0}} e^{-\lambda_{0}\left(L_{0}-t\right)} \frac{\left(L_{0}-t\right)^{S_{0}-1}}{\left(S_{0}-1\right) !}, \quad \text { for } 0 \leq t \leq L_{0} .
$$

The probability mass in the point $Z=0$ is found by using (2),

$$
\mathbf{P}\{Z=0\}=\mathbf{P}\left\{X_{0}>L_{0}\right\}=1-F_{X_{0}}\left(L_{0}\right)=\sum_{n=0}^{S_{0}-1} e^{-\lambda_{0} L_{0}} \frac{\left(\lambda_{0} L_{0}\right)^{n}}{n !}
$$

Let us continue by deriving the probability function for the inventory level at the local site $i, I L_{i}$. Now, given the delay $Z=z$, the conditional stationary probability function for $I L_{i}$ follows as

$$
\mathbf{P}\left\{I L_{i}=k \mid Z=z\right\}=\frac{\left(\lambda_{i}\left(L_{i}+z\right)\right)^{S_{i}-k}}{\left(S_{i}-k\right) !} e^{-\lambda_{i}\left(L_{i}+z\right)},
$$

Using (3)-(5), we may remove the condition on $Z$ as follows

$$
\mathbf{P}\left\{I L_{i}=k\right\}=\frac{\left(\lambda_{i} L_{i}\right)^{S_{i}-k}}{\left(S_{i}-k\right) !} e^{-\lambda_{i} L_{i}} \mathbf{P}\{Z=0\}+\int_{0}^{L_{0}} \mathbf{P}\left\{I L_{i}=k \mid Z=z\right\} f_{Z}(z) d z .
$$

In order to be able to evaluate inventory level probabilities exactly, we provide the following closed form expression of the inventory level probability function:

Proposition 1 For $k \leq S_{i}, S_{0}>0, n:=S_{i}-k, m:=S_{0}-1$, and $\mu:=\lambda_{0}-\lambda_{i} \geq 0$, the closed form expression of $\mathbf{P}\left\{I L_{i}=k\right\}$ is given by

$$
\begin{aligned}
\mathbf{P}\left\{I L_{i}=k\right\}= & \frac{\left(\lambda_{i} L_{i}\right)^{S_{i}-k}}{\left(S_{i}-k\right) !} e^{-\lambda_{i} L_{i}} \mathbf{P}\{Z=0\} \\
& +A \sum_{k_{1}=0}^{n} \sum_{k_{2}=0}^{m}(-1)^{m-k_{2}}\left(\begin{array}{l}
n \\
k_{1}
\end{array}\right)\left(\begin{array}{l}
m \\
k_{2}
\end{array}\right) L_{i}^{k_{1}} L_{0}^{k_{2}} \Psi\left(k_{1}, k_{2}\right),
\end{aligned}
$$


where

$$
\begin{aligned}
\Psi\left(k_{1}, k_{2}\right)= & e^{\mu L_{0}}\left(m+n-k_{1}-k_{2}\right) ! \sum_{j=0}^{m+n-k_{1}-k_{2}}\left[\frac{(-1)^{m+n-k_{1}-k_{2}-j}}{\mu^{m+n-k_{1}-k_{2}-j+1}} \cdot \frac{L_{0}^{j}}{j !}\right] \\
& -\frac{(-1)^{m+n-k_{1}-k_{2}}\left(m+n-k_{1}-k_{2}\right) !}{\mu^{m+n-k_{1}-k_{2}+1}}, \\
A= & \frac{\lambda_{i}^{S_{i}-k}}{\left(S_{i}-k\right) !} \cdot \frac{\lambda_{0}^{S_{0}}}{\left(S_{0}-1\right) !} e^{-\lambda_{i} L_{i}-\lambda_{0} L_{0}} .
\end{aligned}
$$

Proof See "Appendix".

Notice that, Proposition 1 is only defined for $S_{0}>0$. However, for $S_{0}=0$ the problem degenerates to a single-echelon inventory system, i.e., $Z \equiv L_{0}$.

From (7) the average stock on hand at site $i$ is obtained as

$$
\mathbf{E}\left\{I L_{i}^{+}\right\}=\sum_{k=1}^{S_{i}} k \mathbf{P}\left\{I L_{i}=k\right\}
$$

and the average stock on hand at the central warehouse becomes

$$
\mathbf{E}\left\{I L_{0}^{+}\right\}=\sum_{k=1}^{S_{0}} k \frac{\left(\lambda_{0} L_{0}\right)^{S_{0}-k}}{\left(S_{0}-k\right) !} e^{-\lambda_{0} L_{0}}
$$

Let us continue by deriving an expression for the probability that an arriving customer at site $i$ has to wait longer than $\omega_{i}$ units of time. In this case it is reasonable, from a practical point of view, to assume that $0 \leq \omega_{i} \leq L_{i}$. By conditioning on $Z=z$, we obtain the following conditional probability

$$
\begin{aligned}
\mathbf{P}\left\{Y_{i}>\omega_{i} \mid Z=z\right\} & =\mathbf{P}\left\{L_{i}+z-X_{i}>\omega_{i}\right\}=\mathbf{P}\left\{X_{i}<L_{i}+z-\omega_{i}\right\}=F_{X_{i}}\left(L_{i}+z-\omega_{i}\right) \\
& =1-\sum_{n=0}^{S_{i}-1} e^{-\lambda_{i}\left(L_{i}+z-\omega_{i}\right)} \frac{\left(\lambda_{i}\left(L_{i}+z-\omega_{i}\right)\right)^{n}}{n !} .
\end{aligned}
$$

Hence, by using (3), (4), (10) and the law of total probability we obtain

$$
\mathbf{P}\left\{Y_{i}>\omega_{i}\right\}=\mathbf{P}\left\{X_{i}<L_{i}-\omega_{i}\right\} \mathbf{P}\{Z=0\}+\int_{0}^{L_{0}} \mathbf{P}\left\{X_{i}<L_{i}+z-\omega_{i}\right\} f_{Z}(z) d z .
$$

Interestingly enough, similar to Proposition 1, it is possible to derive a closed form expression of the probability in (11).

Proposition 2 The probability that an arriving customer at site $i$ has to wait longer than $\omega_{i}$ units of time is obtained, in closed form, as

$$
\mathbf{P}\left\{Y_{i}>\omega_{i}\right\}=\mathbf{P}\left\{X_{i}<L_{i}-\omega_{i}\right\} \mathbf{P}\{Z=0\}+\mathcal{I},
$$


where

$$
\begin{aligned}
\mathcal{I}= & 1-\mathbf{P}\{Z=0\}-\sum_{n=0}^{S_{i}-1} \Theta(n) \sum_{k_{1}=0}^{n} \sum_{k_{2}=0}^{S_{0}-1}(-1)^{S_{0}-1-k_{2}}\left(\begin{array}{c}
n \\
k_{1}
\end{array}\right)\left(\begin{array}{c}
S_{0}-1 \\
k_{2}
\end{array}\right) \\
& \left(L_{i}-\omega_{i}\right)^{k_{1}} L_{0}^{k_{2}} \Omega\left(n, k_{1}, k_{2}\right), \quad \\
\Omega\left(n, k_{1}, k_{2}\right)= & e^{\left(\lambda_{0}-\lambda_{i}\right) L_{0}}\left(n+S_{0}-1-k_{1}-k_{2}\right) ! \sum_{j=0}^{n+S_{0}-1-k_{1}-k_{2}}\left[\frac{(-1)^{n+S_{0}-1-k_{1}-k_{2}-j}}{\left(\lambda_{0}-\lambda_{i}\right)^{n+S_{0}-k_{1}-k_{2}-j}} \cdot \frac{L_{0}^{j}}{j !}\right] \\
& -\frac{(-1)^{n+S_{0}-1-k_{1}-k_{2}}\left(n+S_{0}-1-k_{1}-k_{2}\right) !}{\left(\lambda_{0}-\lambda_{i}\right)^{n+S_{0}-k_{1}-k_{2}}}, \\
\Theta(n)= & \frac{\lambda_{i}^{n}}{n !} \cdot \frac{\lambda_{0}^{S_{0}}}{\left(S_{0}-1\right) !} e^{-\lambda_{i}\left(L_{i}-\omega\right)-\lambda_{0} L_{0}} .
\end{aligned}
$$

Proof See "Appendix".

\section{Cost structures}

\subsection{Piecewise constant backorder costs}

Assume that a customer arriving at site $i$ incurs a backorder cost that depends on the time the demand is backordered. In more detail, denote $B_{i}\left(Y_{i}\right)$ as the backorder cost, as a function of $Y_{i}$, incurred by a customer arriving at site $i$.

Assume that a customer arriving at site $i$ has an acceptable waiting time of $\omega_{i}$ units of time for a demanded item. As a start, in view of the discussion about service contracts in the introduction, let us consider a particular simple and important case concerning the backorder cost structure:

$$
B_{i}\left(Y_{i}\right)= \begin{cases}0 & \text { if } \quad Y_{i} \leq \omega_{i} \\ b_{i} & \text { if } \quad Y_{i}>\omega_{i}\end{cases}
$$

Hence, if an arriving customer has to wait longer than $\omega_{i}$ for a spare part, then the service providing company is obliged to pay a fixed penalty cost $b_{i}$. Otherwise, no backorder cost is incurred. Notice that, in this setting, the assumption of the FCFS-rule is not optimal but reasonable. For example, say that there are two waiting customers at location $i$ and the customer first in line already has been waiting for more than $\omega_{i}$ units of time, while the second has waited less than $\omega_{i}$. Then, when an item then arrives at location $i$ from the warehouse, it would be more cost efficient to assign the incoming item to the second customer (instead of applying the FCFS-rule). Also, in practice, using a different rule than FCFS could mean that a customer that already has exceeded the waiting time limit would not be prioritized and might end up with a very long waiting time (which in practice would mean that the company soon would be out of business). Similarly, the FCFS-rule is, of course, also an issue for the cases presented in Sects. 4.2 and 4.3.

In a more general case, assume that there are $K$ different time limits, $\omega_{i}^{(j)}, j=1,2, \ldots, K$. We consider the following backorder cost (or penalty cost) structure:

$$
B_{i}\left(Y_{i}\right)= \begin{cases}0 & \text { if } Y_{i} \leq \omega_{i}^{(1)}, \\ b_{i}^{(j)} & \text { if } \omega_{i}^{(j)}<Y_{i} \leq \omega_{i}^{(j+1)}, j=1, \ldots, K-1, \\ b_{i}^{(K)} & \text { if } Y_{i}>\omega_{i}^{(K)} .\end{cases}
$$


That is, if an arriving customer has to wait longer than a prescribed time limit $\omega_{i}^{(j)}$, the service providing company has to pay a fix penalty cost $b_{i}^{(j)}$. Here, we assume that $\omega_{i}^{(1)}<\omega_{i}^{(2)}<\cdots<\omega_{i}^{(K)}$ and $b_{i}^{(1)}<b_{i}^{(2)}<\cdots<b_{i}^{(K)}$, i.e. that the cost is increasing with the customer waiting time.

This cost structure can be extended to also include a standard linear backorder cost per unit of time. However, in this paper we focus on the non-linear cost expressions.

\subsection{Exponential backorder costs}

Here we consider a more general type of non-linear backorder cost structure that depends on the time an arriving customer has to spend in backorder. As mentioned in the introduction, although our solution procedure can handle general non-linear backorder costs, we focus on exponentially increasing backorder costs as a function of the customer waiting time. That is, for $Y_{i}>0$, let us define:

$$
B_{i}\left(Y_{i}\right)=c_{i} \cdot a_{i}^{Y_{i}},
$$

where $a_{i}>1$ and $c_{i}>0, i=1,2, \ldots, N$, are constants. In this case, the backorder cost grows exponentially with the customer's waiting time. This means that the backorder cost rapidly gets large when the customer's waiting time gets longer.

\subsection{Time window service constraint}

Here we consider a case where we replace the backorder cost by a time window service constraint. In more detail, assume that there is an agreement that requires that the service level should be at least $\ell_{i}$ within $\omega_{i}$ units of time. In other words, the time window service level at location $i$ is defined as

$$
\beta_{i}=\mathbf{P}\left\{Y_{i} \leq \omega_{i}\right\} \geq \ell_{i}, \quad \forall i \in\{1, \ldots, N\} .
$$

Notice that, in view of (14), this time window service level can be extended to a more general service level definition where different target service levels may be defined for different intervals of waiting times. For example, immediate service could be $95 \%$, while service within 4 h could be $97 \%$, etc.

\subsection{Evaluation of costs}

For the case where there is a backorder cost, let $E B$ denote the total system expected backorder cost, per unit of time. Then, the expected total cost, $E C$, is obtained as

$$
E C=h_{0} \mathbf{E}\left\{I L_{0}^{+}\right\}+\sum_{i=1}^{N} h_{i} \mathbf{E}\left\{I L_{i}^{+}\right\}+E B,
$$

where $\mathbf{E}\left\{I L_{i}^{+}\right\}$and $\mathbf{E}\left\{I L_{0}^{+}\right\}$are defined in (8) and (9), respectively.

In the following analysis we state explicitly how to evaluate $E B$ for piecewise constant and exponential backorder costs. The cost minimization problem for the case with a time window service constraint is defined and analyzed in the optimization section, see Sect. 5.2.

Piecewise constant backorder costs For the special case of a backorder cost with just one acceptable time limit, $\omega_{i}$, we obtain $E B$ as

$$
E B=\sum_{i=1}^{N} \lambda_{i} b_{i} \mathbf{P}\left\{Y_{i}>\omega_{i}\right\}
$$


For the more general cost structure in (14) it is reasonable to assume that $0 \leq \omega_{i}^{(1)}<$ $\omega_{i}^{(2)}<\cdots<\omega_{i}^{(K)} \leq L_{i}$. Hence, using (11), the probability for a customers time in backorder to be in the interval between two consecutive time limits can be written as

$$
\mathbf{P}\left\{\omega_{i}^{(j)}<Y_{i} \leq \omega_{i}^{(j+1)}\right\}=\mathbf{P}\left\{Y_{i}<\omega_{i}^{(j+1)}\right\}-\mathbf{P}\left\{Y_{i}<\omega_{i}^{(j)}\right\} .
$$

Given these probabilities, the expected backorder cost per unit of time follows as

$$
E B=\sum_{i=1}^{N} \lambda_{i}\left(\sum_{j=1}^{K-1} b_{i}^{(j)} \mathbf{P}\left\{\omega_{i}^{(j)}<Y_{i} \leq \omega_{i}^{(j+1)}\right\}+b_{i}^{(K)} \mathbf{P}\left\{Y_{i}>\omega_{i}^{(K)}\right\}\right) .
$$

Exponential backorder costs Given the cost structure in (15), we derive the expected backorder cost $E B$. The time in backorder, $Y_{i}$, depends both on the age of the oldest item, $X_{i}$, and the delay at the warehouse, $Z$. For a given delay $Z=z$ and age of the oldest item $X_{i}=t$, we have $Y_{i}=L_{i}+z-t$. Hence, the conditional backorder cost for location $i$ becomes

$$
B_{i}\left(Y_{i} \mid X_{i}=t, Z=z\right)=B_{i}\left(L_{i}+z-t\right)=c_{i} \cdot a_{i}^{L_{i}+z-t} .
$$

Using (1) and (20), the conditional expected backorder cost, per unit of time, for location $i$ follows as

$$
E B_{i}(Z=z)=\lambda_{i} \int_{0}^{L_{i}+z} B_{i}\left(L_{i}+z-t\right) f_{X_{i}}(t) d t .
$$

Further, using (3), (4) and (21), we get the (unconditional) expected backorder cost per unit of time for location $i$ as

$$
E B_{i}=E B_{i}(Z=0) \cdot \mathbf{P}\{Z=0\}+\int_{0}^{L_{0}} E B_{i}(Z=z) f_{Z}(z) d z,
$$

which in turn gives us the total expected backorder cost,

$$
E B=\sum_{i=1}^{N} E B_{i}
$$

Remark 1 Observe that when the backorder cost has a linear structure, we have the special case $B_{i}\left(Y_{i} \mid X_{i}=t, Z=z\right)=B_{i}\left(L_{i}+z-t\right)=b_{i}\left(L_{i}+z-t\right)$, which is the backorder cost structure studied in Graves (1985) and Axsäter (1990). This directly implies that

$$
E B_{i}(Z=z)=\lambda_{i} b_{i} \int_{0}^{L_{i}+z}\left(L_{i}+z-t\right) f_{X_{i}}(t) d t .
$$

\section{Optimization}

\subsection{Piecewise constant and general non-linear backorder costs}

For a standard two-echelon inventory system with linear holding and backorder costs, the optimization procedure is relatively simple, see e.g. Axsäter (1990). For example, with linear holding and backorder costs it is easy to show that the total cost function is convex in $S_{i}$, $i=1, \ldots, N$, for a given $S_{0}$. This appealing property is, however, lost when considering non-linear costs, as in our case. Therefore, in our optimization procedure we will use other characteristics of the system when optimizing the base-stock levels. One such characteristic is described in the following remark, which is easy to prove (we omit the details): 
Remark 2 If the leadtime for location $i$ is constant, the holding cost at location $i, H_{i}\left(S_{i}\right)$, is increasing in $S_{i}$. Furthermore, $H_{i}\left(S_{i}\right) \rightarrow \infty$ when $S_{i} \rightarrow \infty$.

Our optimization procedure is based on the property described in Remark 2. Due to the non-convex behavior of the total cost function, we derive upper and lower bounds for the optimal values of $S_{0}$ and $S_{i}, i=1, \ldots, N$, where we denote the optimal value of $S_{i}$ for a given $S_{0}$ as $S_{i}^{*}\left(S_{0}\right)$. Similarly, the overall optimal values of $S_{0}$ and $S_{i}$ are denoted as $S_{0}^{*}$ and $S_{i}^{*}$, respectively. Then, obviously, we have $S_{i}^{*} \leq S_{i}^{*}\left(S_{0}\right) \leq \overline{S_{i}^{*}}$, where $S_{i}^{*}$ and $\overline{S_{i}^{*}}$ are the lower and upper bounds, respectively. In complete analogy, we also derive an upper bound, $\overline{S_{0}^{*}}$, and a lower bound, $S_{0}^{*}$, for the optimal value of $S_{0}$.

Hence, $S_{0}^{*}$ is found by choosing $S_{0} \in\left\{\underline{S_{0}^{*}}, \ldots, \overline{S_{0}^{*}}\right\}$ such that

$$
E C\left(S_{0}\right)=C_{0}\left(S_{0}\right)+\sum_{i=1}^{N} C_{i}\left(S_{0}, S_{i}^{*}\left(S_{0}\right)\right)
$$

is minimized (where $C_{0}(\cdot)$ is the average holding costs per time unit at the warehouse, and $C_{i}(\cdot, \cdot)$ is the average holding and backorder costs at the local site $i$ ).

The computation time regarding the optimization procedure for finding optimal base-stock levels was, on average, quite moderate (in general, less than $1 \mathrm{~min}$ ).

\section{Lower and upper bounds for $S_{i}^{*}$}

Notice that, when $S_{0}=\infty$, the leadtime for location $i$ becomes the shortest possible, i.e., $L_{i}$. Hence, in order to obtain a lower bound for $S_{i}^{*}$, the general idea here is to minimize $C_{i}\left(S_{0}, S_{i}\right)$ with respect to $S_{i}$ given that $S_{0}=\infty$. Now, since $C_{i}\left(S_{0}, S_{i}\right)$, given $S_{0}=\infty$, is not convex in $S_{i}$ we will use Remark 2 for obtaining $S_{i}^{*}:=S_{i}^{*}(\infty)$. Recall that when $S_{0}=\infty$, the leadtime is constant $\left(=L_{i}\right)$ and therefore the characteristics of $H_{i}\left(S_{i}\right)$ in Remark 2 may be used. The method for finding $S_{i}^{*}(\infty)$ can be found below in Algorithm 1. The intuition behind Algorithm 1 is that we continue increasing $S_{i}$ until the holding cost becomes greater than the minimum cost so far. Then, from Remark 1, we know that we can stop the search.

In order to obtain an upper bound for $S_{i}^{*}$, we use exactly the same method as when deriving the corresponding lower bound. Notice that, the longest possible leadtime for location $i$, $L_{0}+L_{i}$, is obtained for $S_{0}=0$. Hence, the difference compared to the derivation of the lower bound is that we, in Algorithm 1, set $S_{0}=0$ when deriving the upper bound for $S_{i}^{*}$. Furthermore, instead of starting the optimization algorithm with $S_{i}=0$, we can here use the lower bound, $S_{i}^{*}$, as a starting point for $S_{i}$.

\section{Optimization of $S_{0}$}

In view of Algorithm 1, the problem of finding the optimal $S_{0}$ is relatively straight-forward. As mentioned, the total cost function is, in general, not convex in $S_{0}$. Therefore, we derive lower and upper bounds for the optimal $S_{0}$. A lower bound for $S_{0}^{*}$ is found by optimizing $E C\left(S_{0}, \overline{S_{1}^{*}}, \overline{S_{2}^{*}}, \ldots, \overline{S_{N}^{*}}\right)$ with respect to $S_{0}$. That is, if all $S_{i}, i=1, \ldots, N$, are fixed and chosen as large as possible, a lower bound for $S_{0}^{*}$ is obtained. The optimization procedure is presented in Algorithm 2 below, and is quite similar to Algorithm 1, with only a few modifications. In complete analogy, an upper bound for $S_{0}^{*}$ is found by optimizing $E C\left(S_{0}, \underline{S_{1}^{*}}, \underline{S_{2}^{*}}, \ldots, \underline{S_{N}^{*}}\right)$ with respect to $S_{0}$. 

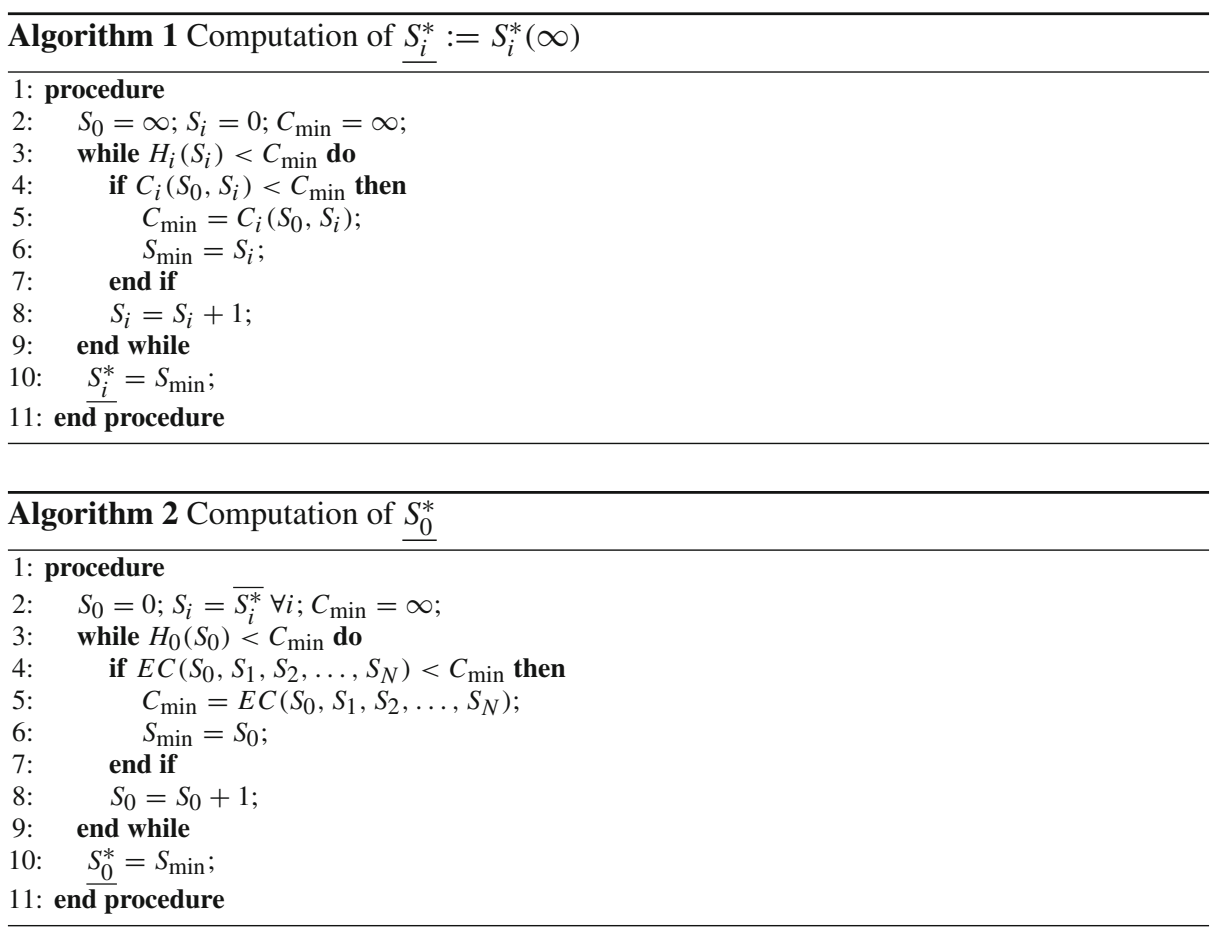

\subsection{Time window service constraint}

The optimization problem, when considering a time window service constraint instead of backorder costs, becomes

$$
\begin{aligned}
& \min _{S_{0} \geq 0, S_{i} \geq 0} \underset{\forall i \in\{1, \ldots, N\}}{ } \operatorname{EC}\left(S_{0}, S_{1}, \ldots, S_{N}\right)=\sum_{i=0}^{N} h_{i} \mathbf{E}\left\{I L_{i}^{+}\right\} \\
& \text {subject to } \beta_{i}=\mathbf{P}\left\{Y_{i} \leq \omega_{i}\right\}=1-\mathbf{P}\left\{Y_{i}>\omega_{i}\right\} \geq \ell_{i}, \quad \forall i \in\{1, \ldots, N\},
\end{aligned}
$$

where $\ell_{i}$ is the target time window service level. The optimization procedure is quite similar as in Sect. 5.1. First, notice that from (10) and (11) it follows that the time window service level $\beta_{i}$ is strictly monotonic in $S_{i}, i \in\{1, \ldots, N\}$, i.e., $\mathbf{P}\left\{Y_{i} \leq \omega_{i} \mid S_{i}\right\}>\mathbf{P}\left\{Y_{i} \leq \omega_{i} \mid S_{i}-1\right\}$. Hence, for a given $S_{0}$, the minimum value of $S_{i}$ which satisfies the time window constraint can easily be found.

In order to find the optimal value of $S_{0}$, again a very similar procedure as in Sect. 5.1 can be developed. In short, given $\overline{S_{i}^{*}}=\arg \min _{S_{i}} C_{i}\left(0, S_{i}\right)$ such that $\beta_{i} \geq \ell_{i}$, a lower bound of the optimal $S_{0}$ becomes 0 since minimizing $\operatorname{EC}\left(S_{0}, \overline{S_{1}^{*}}, \overline{S_{2}^{*}}, \ldots, \overline{S_{N}^{*}}\right)$ with respect to $S_{0}$, such that (25) is satisfied, gives the lower bound $S_{0}^{*}=0$. This is the case since $E C\left(S_{0}, \overline{S_{1}^{*}}, \overline{S_{2}^{*}}, \ldots, \overline{S_{N}^{*}}\right)$ is obviously increasing in $S_{0}$. The upper bound $\overline{S_{0}^{*}}$ can be found in a similar manner by minimizing $E C\left(S_{0}, S_{1}^{*}, S_{2}^{*}, \ldots, \underline{S_{N}^{*}}\right)$ with respect to $S_{0}$, such that (25) is satisfied. That is, we start with $S_{0}=0$ and increase $\overline{S_{0}}$ by one unit at a time until (25) is satisfied. 


\section{Applications and numerical experiments}

In this section we first evaluate the three cases considered in Sect. 4 for a number of test problems. In all test problems we consider an inventory system with two downstream sites, i.e., $N=2$. For the sake of simplicity let us also assume that the local sites are identical (although this is not necessary from a modeling point of view). Secondly, we consider how our model may be used in order to quantify environmental effects associated with not satisfying customer demands in a timely fashion. We provide here a small numerical study concerning sustainability aspects in connection with $\mathrm{CO}_{2}$ emissions.

\subsection{Numerical evaluation of different backorder cost structures}

For the first cost structure with a piecewise constant backorder cost function, we let $K=1$, i.e., there is a single acceptable customer waiting time limit. The customer arrival intensity is either $\lambda_{i}=0.1$ or $\lambda_{i}=0.5$. The holding costs are, for simplicity, assumed to be the same at all locations. In more detail, we consider the settings $h_{0}=h_{i}=0.5$ and $h_{0}=h_{i}=1$. Moreover, we let the backorder cost take values in $b_{i} \in\{10,100,500,1000\}$. The main purpose is to study the effect of the backorder cost in the form of a step function. Notice that, in this numerical study we consider several different ratios of $b_{i}$ and $h_{i}$. A low ratio corresponds to a situation with relatively expensive spare parts. When this ratio is relatively high, the backorder costs are considerably larger than the holding costs. This may be the case if the spare parts are relatively inexpensive and/or it is very costly if the spare part is not available when needed. In all problems the transportation time to the central warehouse is $L_{0}=10$. In Table 1 we have $L_{i}=1$, and in Table 2 we set $L_{i}=5$. The customer's acceptable waiting time is either 10,30 or $50 \%$ of the transportation time to the local sites.

As we can see in Tables 1 and 2, the optimal base-stock levels at the local sites in many cases tend to increase when $\omega_{i}$ gets lower. On the other hand, the optimal base-stock level at the central warehouse tends to, for most cases, decrease with lower $\omega_{i}$. Hence, when the acceptable time limit, $\omega_{i}$, is rather low, inventory should be pushed to the downstream locations. The intuitive explanation is that when $\omega_{i}$ is low, it is relatively likely that an arriving customer's waiting time will exceed $\omega_{i}$ if there is no stock on hand at the downstream sites. Therefore, the base-stock levels at the local sites should be relatively high in these cases. On the other hand, if $\omega_{i}$ is relatively long it is not that crucial to keep stock close to the customers. As expected, the total expected cost increases with higher backorder costs and lower acceptable waiting times.

The results from Tables 1 and 2 also reflect the difficulty of finding structural optimization properties of the system. For example, in Table 1, it is important to notice that the optimal $S_{0}$ is in general not monotonic in $\omega_{i}$. Such characteristics also make it hard for practitioners to develop heuristic optimization procedures which often rely on convexity results. Hence, since the optimization results might be counterintuitive for practitioners, it is important to use the exact optimization procedure developed in Sect. 5. However, it is interesting to note that the optimal total amount of items in the system, $S_{0}^{*}+\sum_{i=1}^{N} S_{i}^{*}$, is non-increasing in $\omega_{i}$ for all cases considered in Tables 1 and 2 . This result is in line with intuition since when $\omega_{i}$ increases, customers acceptable waiting times increase, and we may lower the total amount of stock in the system and still provide sufficiently high customer service. However, due to the non-monotonic property discussed above, it is very difficult to argue exactly how the total stock is allocated among the local sites and the central warehouse.

Apart from the test problems where $b_{i}=10$ in Tables 1 and 2, the probability of exceeding the acceptable time limit is quite small. In these problems the backorder cost, $b_{i}$, is large 


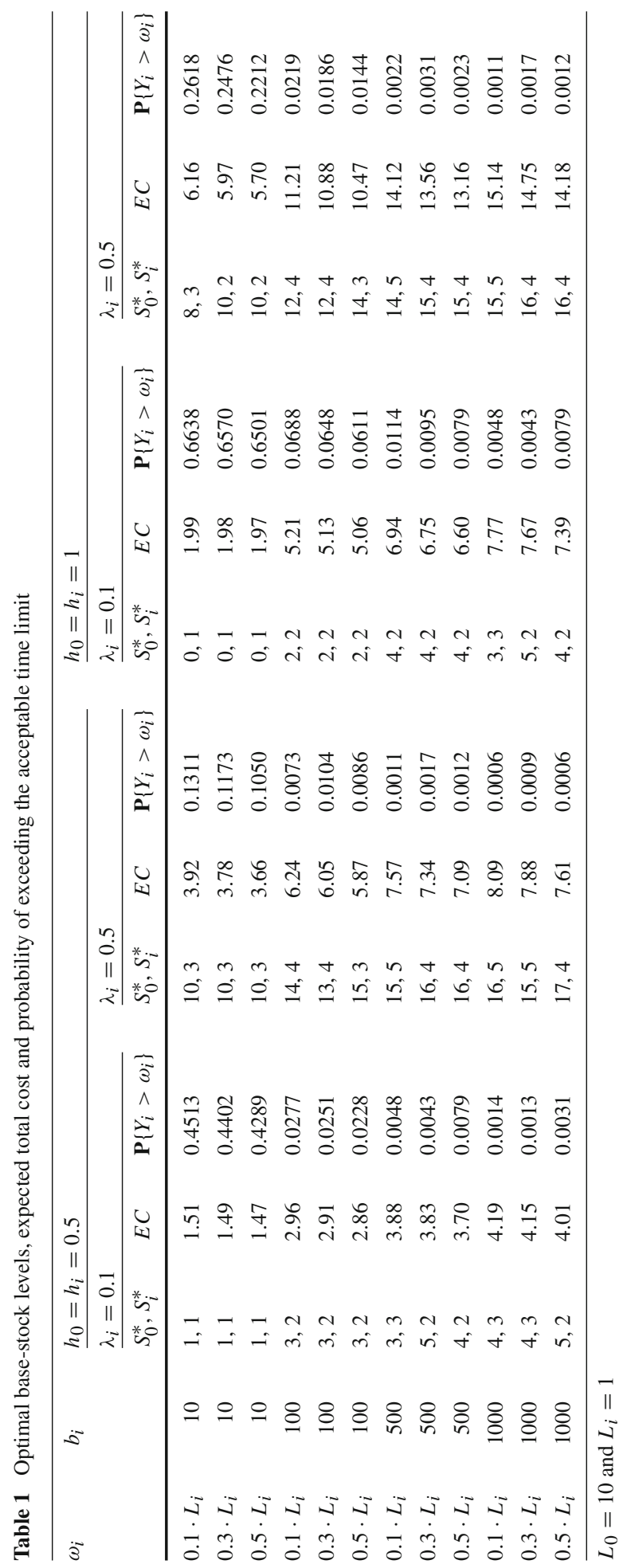




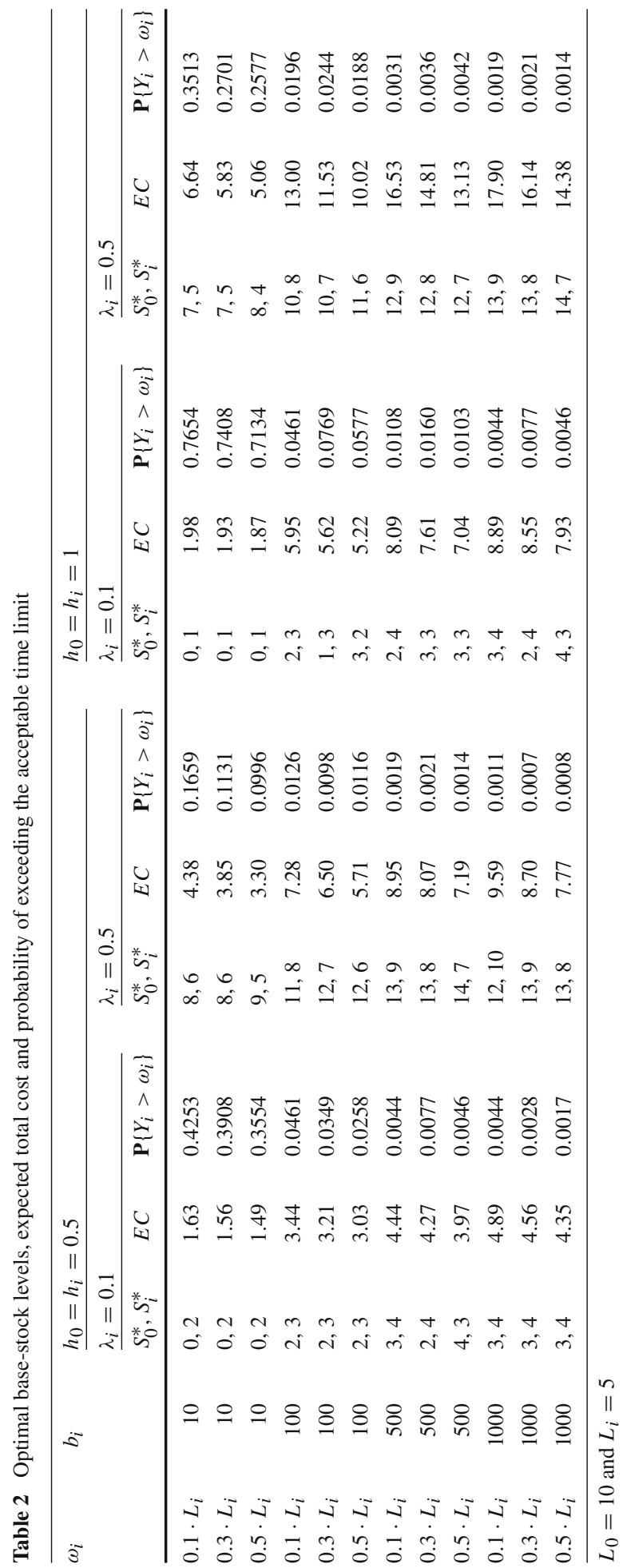


Table 3 Optimal base-stock levels and expected total cost

$L_{0}=10$ and $L_{i}=2$

\begin{tabular}{|c|c|c|c|c|c|c|c|c|}
\hline \multirow[t]{3}{*}{$a_{i}$} & \multicolumn{4}{|c|}{$h_{0}=h_{i}=0.5$} & \multicolumn{4}{|c|}{$\underline{h_{0}=h_{i}}=1$} \\
\hline & \multicolumn{2}{|c|}{$\lambda_{i}=0.1$} & \multicolumn{2}{|c|}{$\lambda_{i}=0.5$} & \multicolumn{2}{|c|}{$\lambda_{i}=0.1$} & \multicolumn{2}{|c|}{$\lambda_{i}=0.5$} \\
\hline & $S_{0}^{*}, S_{i}^{*}$ & $E C$ & $S_{0}^{*}, S_{i}^{*}$ & $E C$ & $S_{0}^{*}, S_{i}^{*}$ & $E C$ & $S_{0}^{*}, S_{i}^{*}$ & $E C$ \\
\hline 1.1 & 1,0 & 0.50 & 6,2 & 1.48 & 1,0 & 0.57 & 6,1 & 1.75 \\
\hline 1.5 & 2,1 & 1.48 & 9,3 & 2.64 & 3,0 & 2.40 & 9,2 & 3.93 \\
\hline 2 & 4,1 & 2.15 & 11,3 & 3.51 & 3,1 & 3.91 & 10,3 & 5.88 \\
\hline 4 & 7,1 & 3.64 & 15,3 & 5.38 & 6,1 & 6.75 & 14,3 & 9.85 \\
\hline
\end{tabular}

compared to the holding cost $h_{i}$. Moreover, as expected, we see that the probability of exceeding the acceptable time limit is significantly higher when the backorder cost tends to be relatively low. When the items are relatively expensive to keep in stock we note that the optimal base-stock levels are low, especially when the customer arrival rate is low.

For our second cost structure with an exponentially increasing backorder cost function, we evaluate our model for a set of test problems where we let $c_{i}=1$ and $a_{i}$ in (15) be one of four values, $a_{i} \in\{1.1,1.5,2,4\}$. As before, the customer arrival rate is either $\lambda_{i}=0.1$ or $\lambda_{i}=0.5$. The holding costs are the same at all locations and either $h_{0}=h_{i}=0.5$ or $h_{0}=h_{i}=1$. The transportation times are $L_{0}=10$ and $L_{i}=2$. We consider different ratios of $a_{i}$ and $h_{i}$ where a lower ratio corresponds to relatively expensive spare parts and vice versa.

As seen in Table 3, the optimal base-stock levels at the central warehouse increase rather rapidly with increasing values of $a_{i}$. It is also interesting to notice that the base-stock levels at the local sites are kept at relatively low levels, although $a_{i}$ increases significantly. The intuition behind this behavior is that when $a_{i}$ is relatively large, long customer waiting times will be very costly, while short waiting times are not so expensive. Notice that, in order to suppress long customer waiting times it may be wise to allocate stock to the central warehouse instead of the local sites. That is, instead of allocating relatively large amount of stock to each local site, the optimal stock policy will suggest that stock should be kept upstream, since long waiting times can be suppressed just as well from stock at the central warehouse. It is interesting to note that this result runs absolutely counter to traditional multi-echelon models with fill rate constraints, see e.g., Muckstadt and Thomas (1980), Axsäter (2003a), and Hausman and Erkip (1994). Optimal solutions of such inventory systems very often show that inventory should be prioritized downstream, while the fill rate at the central warehouse may be only about $50 \%$.

For the case with time window service level constraints $\beta_{i} \geq \ell_{i}$, we evaluate our model for test problems with three different levels of the time window service level that must be achieved, that is $\ell_{i} \in\{0.90,0.95,0.98\}$. As before, the customer arrival rate is either $\lambda_{i}=0.1$ or $\lambda_{i}=0.5$. The holding costs are the same at all locations and either $h_{0}=h_{i}=0.5$ or $h_{0}=h_{i}=1$. The transportation times are $L_{0}=10$ and $L_{i}=2$ and the acceptable waiting time $\omega_{i}$ is either $0,10,30$ or $50 \%$ of the transportation time $L_{i}$.

In Table 4, we see that there often is little or no change in the optimal base-stock levels depending on the value of $\omega_{i}$. With increasing $\ell_{i}$, the base-stock levels also increase, as expected. Notice that, a time window service level constraint where $\omega_{i}=0$ is the same as the traditional fill rate defined as the fraction of demand that can be satisfied immediately from stock on hand. Comparing the test problems in Table 4 where $\omega_{i}$ is zero to the the ones where $\omega_{i}$ has a larger value, it can be seen that optimizing the system using the traditional fill rate even though customers are willing to wait for a certain amount of time may lead to increased costs, especially for higher holding costs or higher customer arrival rates. For 


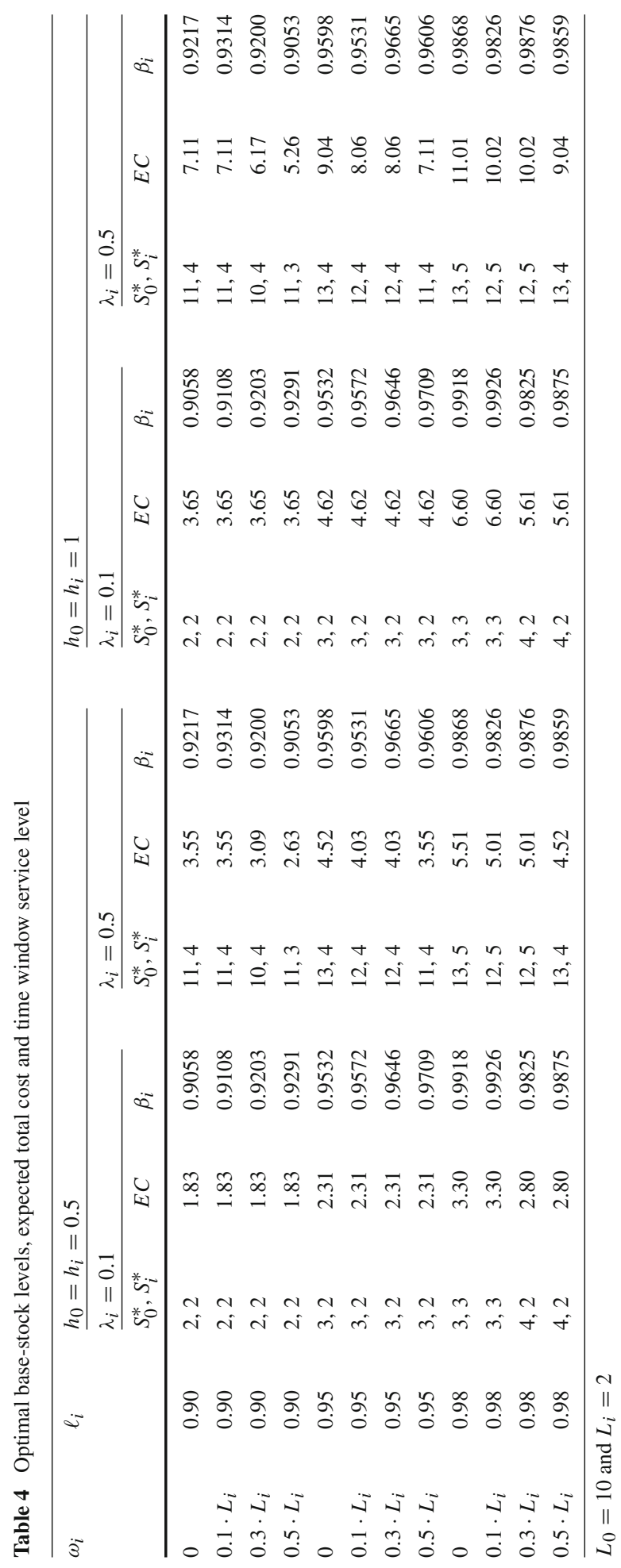


example, among the test problems, the cost increase can be as high as 35\% (see the problems where $h_{i}=1, \lambda_{i}=0.5$, and $\left.\omega_{i}=0.5 L_{i}\right)$.

\subsection{Application: sustainable inventory control}

Let us in this section demonstrate how our model may be used for quantifying $\mathrm{CO}_{2}$ emissions emanating from the customer production site. To this end, we focus on the practical case concerning packaging of dairy products, as mentioned in Sect. 1. To be more specific, let us assume that the dairy product is milk. Quite a few previous studies have investigated the carbon footprint related to milk production. Thoma et al. (2013) conclude that the range of $\mathrm{CO}_{2}$ emissions is approximately $0.75-1.5 \mathrm{~kg}$, per kg milk produced. Hence, we assume that, on average, there is a one to one correspondence between the amount of milk produced and the amount of $\mathrm{CO}_{2}$ emissions (in units of weight).

Recall from the discussion in Sect. 1 that we assume a whole milk-batch is wasted if the production downtime exceeds the critical time limit $\omega_{i}$. Hence, the expected $\mathrm{CO}_{2}$ emissions of production waste in $\mathrm{kg}$ per unit of time becomes

$$
\mathbf{E}\left\{\mathrm{CO}_{2}\right\}=\sum_{i=1}^{N} \lambda_{i} \mathbf{P}\left\{Y_{i}>\omega_{i}\right\} M_{i},
$$

where $M_{i}$ is the average batch size (in $\mathrm{kg}$ ) at the production facility corresponding to the local inventory site $i$. In practice, $M_{i}$ ranges from approximately $1000-30,000 \mathrm{~kg}$.

Similar expressions for $\mathbf{E}\left\{\mathrm{CO}_{2}\right\}$ for other products than milk may, of course, be evaluated by using exactly the same modeling technique, and the evaluation of $\mathbf{E}\left\{\mathrm{CO}_{2}\right\}$ in (26) may be of interest in many other related applications. For example, if a $\mathrm{CO}_{2}$ tax is introduced by the government for the specific product produced, a corresponding model should take the average $\mathrm{CO}_{2}$ cost into account. Another related problem is government imposed restrictions on $\mathrm{CO}_{2}$ emissions for specific products. In such a case, our modeling technique may be used in order to evaluate if these $\mathrm{CO}_{2}$ restrictions are satisfied or not, when deciding inventory target levels in spare part logistics systems.

In order to set the $\mathrm{CO}_{2}$ emissions emanating from customer production sites in relation to $\mathrm{CO}_{2}$ emissions from transportation, let us consider a small set of numerical examples. For simplicity, we consider the same test bed as in Table 1. That is, in Table 1, we present optimal base-stock levels and probabilities $\mathbf{P}\left\{Y_{i}>\omega_{i}\right\}$ for a system with $N=2$ locations (for a specific parameter setting). Assuming $M_{i}=15,000$ as a benchmark, and given the probabilities $\mathbf{P}\left\{Y_{i}>\omega_{i}\right\}$ in Table 1 (for the case $h_{0}=h_{i}=1$ ) we list, in Table 5, the corresponding expected $\mathrm{CO}_{2}$ emissions of production waste. As mentioned, to set the $\mathrm{CO}_{2}$ emissions of production waste in relation to transportation, it is interesting to notice that the carbon intensity (expressed as $\mathrm{gCO}_{2}$ per tonne-km) for heavy trucks is approximately 200 (McKinnon 2010). This corresponds to 5 tonne-km per $\mathrm{kg} \mathrm{CO}_{2}$. To illustrate this relation we also list, in Table 5, the equivalent number of tonne-km of transportation by heavy trucks.

In Table 5, we can conclude that $\mathbf{E}\left\{\mathrm{CO}_{2}\right\}$ may be relatively large even for quite low probabilities of exceeding the acceptable waiting time. Of course, it is also clear that when $\mathbf{P}\left\{Y_{i}>\omega_{i}\right\}$ is very low (which corresponds to a very high fixed penalty cost), then $\mathbf{E}\left\{\mathrm{CO}_{2}\right\}$ is also very low. However, it is important to realize that in some situations the $\mathrm{CO}_{2}$ emissions related to (too long) production downtime may be relatively high, while the downtime cost may be moderate. For example, in order to avoid discarding a dairy product, excessive energy in terms of cooling may be considered (for other types of products, excessive heating has to be initiated in order to avoid obsolescence). 
Table 5 Examples of $\mathbf{E}\left\{\mathrm{CO}_{2}\right\}$ and the corresponding number of tonne-km of transportation by heavy trucks

\begin{tabular}{|c|c|c|c|c|c|}
\hline \multicolumn{3}{|l|}{$\lambda_{i}=0.1$} & \multicolumn{3}{|l|}{$\lambda_{i}=0.5$} \\
\hline $\mathbf{P}\left\{Y_{i}>\omega_{i}\right\}$ & $\mathbf{E}\left\{\mathrm{CO}_{2}\right\}(\mathrm{kg})$ & Transportation $(\mathrm{km})$ & $\mathbf{P}\left\{Y_{i}>\omega_{i}\right\}$ & $\mathbf{E}\left\{\mathrm{CO}_{2}\right\}$ & Transportation $(\mathrm{km})$ \\
\hline 0.6638 & 1991 & 9955 & 0.2618 & 3927 & 19,635 \\
\hline 0.6570 & 1971 & 9855 & 0.2476 & 3714 & 18,570 \\
\hline 0.6501 & 1950 & 9750 & 0.2212 & 3318 & 16,590 \\
\hline 0.0688 & 206 & 1030 & 0.0219 & 329 & 1645 \\
\hline 0.0648 & 194 & 970 & 0.0186 & 279 & 1395 \\
\hline 0.0611 & 183 & 915 & 0.0144 & 216 & 1080 \\
\hline 0.0114 & 34 & 170 & 0.0022 & 33 & 165 \\
\hline 0.0095 & 28 & 140 & 0.0031 & 47 & 235 \\
\hline 0.0079 & 24 & 120 & 0.0023 & 36 & 180 \\
\hline 0.0048 & 14 & 70 & 0.0011 & 17 & 85 \\
\hline 0.0043 & 13 & 65 & 0.0017 & 26 & 130 \\
\hline 0.0079 & 24 & 120 & 0.0012 & 18 & 90 \\
\hline
\end{tabular}

\section{Conclusions}

In this paper, we have presented an exact analysis of a two-echelon spare part inventory model with new types of backorder cost structures. By using the stationary age distribution of the units in the system we extended the results from Graves (1985) and Axsäter (1990) by deriving exact closed form expressions for the inventory level distributions (Proposition 1), and also for the customer waiting time distributions (Proposition 2).

Furthermore, we analyzed a model with a piecewise constant backorder cost, where a significant fixed cost is incurred if the customer waiting time exceeds a pre-specified threshold value. This backorder cost structure was then generalized to a general non-linear backorder cost as a function of the customer waiting time. As an example, we analyzed a model with an exponentially increasing backorder cost as a function of the waiting time. Moreover, a corresponding model with time window service levels was explored. As a final step, we investigated how policy decisions affect, indirectly, the expected $\mathrm{CO}_{2}$ emissions related to production waste. These kinds of indirect consequences of policy decisions, in terms of expected $\mathrm{CO}_{2}$ emissions, have been largely ignored in the literature. Instead, most papers have focused on $\mathrm{CO}_{2}$ emissions from a transportation point of view.

For all cases considered in this paper, we also developed optimization procedures for the base-stock levels. Using these optimization procedures, we presented a numerical study in order to investigate how the optimal policy (given the base-stock policy structure) behaves. In particular, contrary to most results from multi-echelon inventory models, it is interesting to notice that inventory should be pushed to the central warehouse in cases where the cost for long waiting times are significantly larger than for short ones.

Possible future extensions may be to include some kinds of emergency supply in order to avoid stops in production and production waste. Another line of research would be to generalize to more complex demand structures, such as compound Poisson demand. Such an extension would be rather straight-forward by keeping track of all individual items in an ordered batch. Admittedly, the computational effort associated with obtaining exact inventory level distributions would, however, be significant. 
Acknowledgements The authors thank two anonymous referees and Professor Johan Marklund for the useful remarks and suggestions.

Open Access This article is distributed under the terms of the Creative Commons Attribution 4.0 International License (http://creativecommons.org/licenses/by/4.0/), which permits unrestricted use, distribution, and reproduction in any medium, provided you give appropriate credit to the original author(s) and the source, provide a link to the Creative Commons license, and indicate if changes were made.

\section{Appendix}

Proof of Proposition 1 Consider the integral in (6). We have:

$$
\begin{aligned}
& \int_{0}^{L_{0}} \mathbf{P}\left\{I L_{i}=k \mid Z=z\right\} f_{Z}(z) d z=\int_{0}^{L_{0}} \frac{\left(\lambda_{i}\left(L_{i}+z\right)\right)^{S_{i}-k}}{\left(S_{i}-k\right) !} e^{-\lambda_{i}\left(L_{i}+z\right)} \\
& \cdot \lambda_{0}^{S_{0}} e^{-\lambda_{0}\left(L_{0}-z\right)} \frac{\left(L_{0}-z\right)^{S_{0}-1}}{\left(S_{0}-1\right) !} d z=\frac{\lambda_{i}^{S_{i}-k}}{\left(S_{i}-k\right) !} \cdot \frac{\lambda_{0}^{S_{0}}}{\left(S_{0}-1\right) !} e^{-\lambda_{i} L_{i}-\lambda_{0} L_{0}} \\
& \int_{0}^{L_{0}}\left(L_{i}+z\right)^{S_{i}-k}\left(L_{0}-z\right)^{S_{0}-1} e^{\left(\lambda_{0}-\lambda_{i}\right) z} d z
\end{aligned}
$$

Now, for notational purposes we set $n:=S_{i}-k, m:=S_{0}-1$, and $\mu:=\lambda_{0}-\lambda_{i} \geq 0$. Then, the well known binomial theorem (see any textbook in Calculus) gives,

$$
\left(L_{i}+z\right)^{n}\left(L_{0}-z\right)^{m}=\sum_{k_{1}=0}^{n} \sum_{k_{2}=0}^{m}\left(\begin{array}{l}
n \\
k_{1}
\end{array}\right) L_{i}^{k_{1}} z^{n-k_{1}}\left(\begin{array}{l}
m \\
k_{2}
\end{array}\right) L_{0}^{k_{2}}(-z)^{m-k_{2}} .
$$

Hence, we have the integral

$$
\begin{aligned}
& A \int_{0}^{L_{0}}\left(L_{i}+z\right)^{S_{i}-k}\left(L_{0}-z\right)^{S_{0}-1} e^{\mu z} d z \\
& =A \int_{0}^{L_{0}}\left(\sum_{k_{1}=0}^{n} \sum_{k_{2}=0}^{m}\left(\begin{array}{c}
n \\
k_{1}
\end{array}\right) L_{i}^{k_{1}} z^{n-k_{1}}\left(\begin{array}{l}
m \\
k_{2}
\end{array}\right) L_{0}^{k_{2}}(-z)^{m-k_{2}}\right) e^{\mu z} d z \\
& =A \sum_{k_{1}=0}^{n} \sum_{k_{2}=0}^{m}(-1)^{m-k_{2}}\left(\begin{array}{l}
n \\
k_{1}
\end{array}\right)\left(\begin{array}{l}
m \\
k_{2}
\end{array}\right) L_{i}^{k_{1}} L_{0}^{k_{2}} \int_{0}^{L_{0}} z^{m+n-k_{1}-k_{2}} e^{\mu z} d z,
\end{aligned}
$$

where

$$
A=\frac{\lambda_{i}^{S_{i}-k}}{\left(S_{i}-k\right) !} \cdot \frac{\lambda_{0}^{S_{0}}}{\left(S_{0}-1\right) !} e^{-\lambda_{i} L_{i}-\lambda_{0} L_{0}}
$$

What remains is to calculate the integral $\Psi=\int_{0}^{L_{0}} z^{m+n-k_{1}-k_{2}} e^{\mu z} d z$. For this task, let us first consider an arbitrary positive integer $M$ and a constant $a \geq 0$. Then, by successive integration by parts we obtain the following indefinite integral

$$
\int z^{M} e^{a z} d z=\frac{z^{M}}{a} e^{a z}-\frac{M}{a} \int z^{M-1} e^{a z} d z=\cdots=e^{a z} M ! \sum_{j=0}^{M} \frac{(-1)^{M-j}}{a^{M-j+1}} \cdot \frac{z^{j}}{j !} .
$$


Hence, by using (27), we have the following definite integral

$$
\begin{aligned}
\Psi= & \int_{0}^{L_{0}} z^{m+n-k_{1}-k_{2}} e^{\mu z} d z \\
= & e^{\mu L_{0}}\left(m+n-k_{1}-k_{2}\right) ! \sum_{j=0}^{m+n-k_{1}-k_{2}}\left[\frac{(-1)^{m+n-k_{1}-k_{2}-j}}{\mu^{m+n-k_{1}-k_{2}-j+1}} \cdot \frac{L_{0}^{j}}{j !}\right] \\
& -\frac{(-1)^{m+n-k_{1}-k_{2}}\left(m+n-k_{1}-k_{2}\right) !}{\mu^{m+n-k_{1}-k_{2}+1}} .
\end{aligned}
$$

To conclude,

$$
\mathbf{P}\left\{I L_{i}=k\right\}=\frac{\left(\lambda_{i} L_{i}\right)^{S_{i}-k}}{\left(S_{i}-k\right) !} e^{-\lambda_{i} L_{i}} \mathbf{P}\{Z=0\}+A \sum_{k_{1}=0}^{n} \sum_{k_{2}=0}^{m}(-1)^{m-k_{2}}\left(\begin{array}{l}
n \\
k_{1}
\end{array}\right)\left(\begin{array}{l}
m \\
k_{2}
\end{array}\right) L_{i}^{k_{1}} L_{0}^{k_{2}} \Psi .
$$

Proof of Proposition 2 Consider the integral in (11), and let us once again define $\mu:=$ $\lambda_{0}-\lambda_{i} \geq 0$. Denoting this integral as $\mathcal{I}$, we get:

$$
\begin{aligned}
\mathcal{I} & =\int_{0}^{L_{0}} \mathbf{P}\left\{X_{i}<L_{i}+z-\omega_{i}\right\} f_{Z}(z) d z \\
& =\int_{0}^{L_{0}}\left(1-\sum_{n=0}^{S_{i}-1} e^{-\lambda_{i}\left(L_{i}+z-\omega_{i}\right)} \frac{\left(\lambda_{i}\left(L_{i}+z-\omega_{i}\right)\right)^{n}}{n !}\right) \cdot \lambda_{0}^{S_{0}} e^{-\lambda_{0}\left(L_{0}-z\right)} \frac{\left(L_{0}-z\right)^{S_{0}-1}}{\left(S_{0}-1\right) !} d z \\
& =\int_{0}^{L_{0}} f_{Z}(z) d z-\int_{0}^{L_{0}} \sum_{n=0}^{S_{i}-1} \frac{\lambda_{i}^{n}}{n !} \cdot \frac{\lambda_{0}^{S_{0}}}{\left(S_{0}-1\right) !} e^{-\lambda_{i}\left(L_{i}-\omega\right)-\lambda_{0} L_{0}}\left(L_{i}+z-\omega_{i}\right)^{n}\left(L_{0}-z\right)^{S_{0}-1} e^{\mu z} d z .
\end{aligned}
$$

By defining the function $\Theta(n)$ as,

$$
\Theta(n)=\frac{\lambda_{i}^{n}}{n !} \cdot \frac{\lambda_{0}^{S_{0}}}{\left(S_{0}-1\right) !} e^{-\lambda_{i}\left(L_{i}-\omega\right)-\lambda_{0} L_{0}},
$$

we obtain a similar solution as in the proof of Proposition 1:

$$
\begin{aligned}
\mathcal{I}= & 1-\mathbf{P}\{Z=0\}-\sum_{n=0}^{S_{i}-1} \Theta(n) \int_{0}^{L_{0}}\left(L_{i}+z-\omega_{i}\right)^{n}\left(L_{0}-z\right)^{S_{0}-1} e^{\mu z} d z \\
= & 1-\mathbf{P}\{Z=0\}-\sum_{n=0}^{S_{i}-1} \Theta(n)\left(\sum_{k_{1}=0}^{n} \sum_{k_{2}=0}^{S_{0}-1}(-1)^{S_{0}-1-k_{2}}\left(\begin{array}{c}
n \\
k_{1}
\end{array}\right)\left(\begin{array}{c}
S_{0}-1 \\
k_{2}
\end{array}\right)\left(L_{i}-\omega_{i}\right)^{k_{1}} L_{0}^{k_{2}}\right. \\
& \left.\cdot \int_{0}^{L_{0}} z^{n+S_{0}-1-k_{1}-k_{2}} e^{\mu z} d z\right) .
\end{aligned}
$$

By using (27), the integral in (28) becomes

$$
\begin{aligned}
& \int_{0}^{L_{0}} z^{n+S_{0}-1-k_{1}-k_{2}} e^{\mu z} d z=e^{\mu L_{0}}\left(n+S_{0}-1-k_{1}-k_{2}\right) ! \\
& \quad \sum_{j=0}^{n+S_{0}-1-k_{1}-k_{2}}\left[\frac{(-1)^{n+S_{0}-1-k_{1}-k_{2}-j}}{\mu^{n+S_{0}-k_{1}-k_{2}-j}} \cdot \frac{L_{0}^{j}}{j !}\right]-\frac{(-1)^{n+S_{0}-1-k_{1}-k_{2}}\left(n+S_{0}-1-k_{1}-k_{2}\right) !}{\mu^{n+S_{0}-k_{1}-k_{2}}} .
\end{aligned}
$$


To conclude,

$$
\mathbf{P}\left\{Y_{i}>\omega_{i}\right\}=\mathbf{P}\left\{X_{i}<L_{i}-\omega_{i}\right\} \mathbf{P}\{Z=0\}+\mathcal{I} .
$$

\section{References}

Alkawaleet, N., Hsieh, Y., \& Wang, Y. (2014). Inventory routing problem with $\mathrm{CO}_{2}$ emissions consideration. In P. Golinska (Ed.), Logistics operations, supply chain management and sustainability. Switzerland: Springer.

Axsäter, S. (1990). Simple solution procedures for a class of two-echelon inventory problems. Operations Research, 38, 64-69.

Axsäter, S. (1993). Continuous review policies for multi-level inventory systems with stochastic demand. In S. C. Graves, et al. (Eds.), Handbooks in $O R \& M S$ (Vol. 4, pp. 175-197). Amsterdam: North Holland.

Axsäter, S. (2000). Exact analysis of continuous review $(R, Q)$ policies in two-echelon inventory systems with compound Poisson demand. Operations Research, 48, 686-696.

Axsäter, S. (2003a). Supply chain operations: Serial and distribution inventory systems. In A. G. de Kok, S. Graves (Eds.), Handbooks in OR \& MS, Vol. 11, supply chain management: Design, coordination and operations. Amsterdam: Elsevier.

Axsäter, S. (2003b). A new decision rule for lateral transshipments in inventory systems. Management Science, 49, 1168-1179.

Basten, R. J. I., \& van Houtum, G. J. (2014). System-oriented inventory models for spare parts. Surveys in Operations Research and Management Science, 19, 34-55.

Caggiano, K. E., Muckstadt, P. L., \& Rappold, J. A. (2007). Optimizing service parts inventory in a multiechelon, multi-item supply chain with time-based customer service-level agreements. Operations Research, 55, 303-318.

Cohen, M. A., Agrawal, N., \& Agrawal, V. (2006). Winning the aftermarket. Harvard Business Review, 84, 129-138.

Ettl, M., Feigin, G. E., Lin, G. Y., \& Yao, D. D. (2000). A supply network model with base-stock control and service requirements. Operations Research, 48, 216-232.

Graves, S. C. (1985). A multi-echelon inventory model for a repairable item with one-for-one replenishment. Management Science, 31, 1247-1256.

Harrington, L. (2007). From just in case to just in time. Air Transport World, 4, 77-80.

Hausman, W. H., \& Erkip, N. K. (1994). Multi-echelon vs. single-echelon inventory control policies for low-demand items. Management Science, 40, 597-602.

Howard, C., Marklund, J., Tan, T., \& Reijnen, I. (2015). Inventory control in a spare parts distribution system with emergency stocks and pipeline information. Manufacturing \& Service Operations Management, 17 , $142-156$.

Huang, S., Axsäter, S., Dou, Y., \& Chen, J. (2011). A real-time decision rule for an inventory system with committed service time and emergency orders. European Journal of Operational Research, 215, 70-79.

Katehakis, M. N., \& Smit, L. C. (2012). On computing optimal $(Q, r)$ replenishment policies under quantity discounts. Annals of Operations Research, 200, 279-298.

Marklund, J., \& Berling, P. (2017). Green inventory management. In Y. Bouchery, T. Tan, J. Fransoo, \& C. Corbett (Eds.), Sustainable supply chains. Berlin: Springer.

McKinnon, A. (2010). Green logistics: The carbon agenda. LogForum, 6, 1-9.

Moinzadeh, K., \& Aggarwal, P. K. (1997). An information based multiechelon inventory system with emergency orders. Operations Research, 45, 694-701.

Moinzadeh, K., \& Schmidt, C. P. (1991). An $(S-1, S)$ inventory system with emergency orders. Operations Research, 39, 308-321.

Muckstadt, J. A., \& Thomas, L. J. (1980). Are multi-echelon inventory models worth implementing in systems with low-demand-rate items? Management Science, 26, 483-494.

Olsson, F. (2015). Emergency lateral transshipments in a two-echelon inventory system with positive transshipment leadtimes. European Journal of Operational Research, 242, 424-433.

Paterson, C., Kiesmüller, G., Teunter, R., \& Glazebrook, K. (2011). Inventory models with lateral transshipments: A review. European Journal of Operational Research, 210, 125-136.

Sherbrooke, C. C. (1968). METRIC: A multi-echelon technique for recoverable item control. Operations Research, 16, 122-141. 
Thoma, G., Popp, J., Nutter, D., Shonnard, D., Ulrich, R., Matlock, M., et al. (2013). Greenhouse gas emissions from milk production and consumption in the United States: A cradle-to-grave life cycle assessment circa 2008. International Dairy Journal, 31, S3-S14.

Turban, E. (1988). Review of expert systems technology. IEEE Transactions on Engineering Management, 35, 71-81.

US Department of Commerce, Office of Transportation and Machinery (2009). US automotive parts industry annual assessment.

Wong, H., Kranenburg, B., van Houtum, G. J., \& Cattrysse, D. (2007). Efficient heuristics for two-echelon spare parts inventory systems with an aggregate mean waiting time constraint per local warehouse. OR-Spectrum, 29, 699-722.

Yang, G., Dekker, R., Gabor, A. F., \& Axsäter, S. (2013). Service parts inventory control with lateral transshipment and pipeline stock flexibility. International Journal of Production Economics, 142, 278-289. 